\title{
Two-Phase Flow in Porous Media: Dynamic Capillarity and Heterogeneous Media
}

\author{
C. J. van Duijn ${ }^{1} \cdot$ X. Cao ${ }^{1}$ - I. S. Pop ${ }^{1,2}$
}

Received: 8 April 2015 / Accepted: 7 July 2015 / Published online: 11 August 2015

(C) The Author(s) 2015. This article is published with open access at Springerlink.com

\begin{abstract}
We investigate a two-phase porous media flow model, in which dynamic effects are taken into account in phase pressure difference. We consider a one-dimensional heterogeneous case, with two adjacent homogeneous blocks separated by an interface. The absolute permeability is assumed constant, but different in each block. This may lead to the entrapment of the non-wetting phase (say, oil) when flowing from the coarse material into the fine material. We derive the interface conditions coupling the models in each homogeneous block. In doing so, the interface is approximated by a thin porous layer, and its thickness is then passed to zero. Such results have been obtained earlier for standard models, based on equilibrium relationship between the capillary pressure and the saturation. Then, oil is trapped until its saturation on the coarse material side of the interface exceeds an entry value. In the non-equilibrium case, the situation is different. Due to the dynamic effects, oil may still flow into the fine material even after the saturation drops under the entry point, and this flow may continue for a certain amount of time that is proportional to the non-equilibrium effects. This suggests that operating in a dynamic regime reduces the account of oil trapped at interfaces, leading to an enhanced oil recovery. Finally, we present some numerical results supporting the theoretical findings.
\end{abstract}

Keywords Dynamic capillary pressure $\cdot$ Immiscible flow $\cdot$ Heterogeneity $\cdot$ Trapping

$凶$ X. Cao

x.cao@tue.nl

C. J. van Duijn

c.j.v.duijn@tue.nl

I. S. Pop

i.pop@tue.nl

1 CASA, Eindhoven University of Technology, P.O. Box 513, 5600 MB Eindhoven, The Netherlands

2 Department of Mathematics, University of Bergen, P.O. Box 7800, 5020 Bergen, Norway 


\section{Introduction}

Two-phase (wetting/non-wetting) flows are widely encountered in various real-life processes. A few prominent examples are water-driven oil recovery, or geological sequestration of $\mathrm{CO}_{2}$. These processes involve large spatial scales, and (rock) heterogeneities appear naturally. In this paper, we consider a simplified situation, where the medium consists of two different homogeneous blocks with different permeabilities (coarse and fine), which are separated by an interface. This makes the transition from one material to another not smooth, and appropriate conditions have to be imposed at the interface for coupling the models written in each of the two blocks. In particular, when the underlying models are involving entry pressures to describe the dependency of the capillary pressure on the phase saturations, the non-wetting phase may remain trapped in the coarse block at the interface.

This situation has been analyzed in Duijn et al. (1995) and Duijn and Neef (1998), but for the case when the phase pressure difference depends on the, say, wetting phase saturation and the medium itself. These are standard models, for which the dependency between various quantities are determined under equilibrium conditions. Therefore, such models are also called equilibrium models. In the paper mentioned above, regularization arguments (i.e., approximating the interface by a thin porous layer ensuring a smooth transition between the two homogeneous blocks) are employed to derive appropriate coupling conditions between the models in the two sub-domains. The resulting conditions are the flux continuity and an extended pressure condition. We refer to Bertsch et al. (2003), Buzzi et al. (2009) and Cancès and Pierre (2012) for the mathematical analysis of such models, where the existence of weak solutions has been analyzed. Further, the case of many layers is studied in Duijn et al. (2002, 2007), where homogenization techniques are applied for deriving an effective model. Such kind of models are also studied in Andreianov and Cancès (2014), Brenner et al. (2013), Fučík and Mikyška (2011), Fučík and Mikyška (2011), Radu (2004) and Radu et al. (2015), where appropriate numerical schemes are studied.

Various experiments Bottero (2009) and DiCarlo (2004) have invalidated the equilibrium assumptions, and motivated considering non-equilibrium approaches. Here, we focus on models involving dynamic effects in the phase pressure difference, as proposed in Hassanizadeh and Gray (1993). In this case, we follow the ideas in Duijn et al. (1995) and Duijn and Neef (1998) and derive the coupling conditions at the interface separating the two homogeneous blocks. When compared to the equilibrium case, a striking difference appears. In the former, the non-wetting phase can only flow into the fine block if its saturation at the coarse block side of the interface exceeds an entry value, and in the latter situation, this flow can appear for lower saturation values. This is due to the dynamic effects in the phase pressure difference and can reduce the amount of non-wetting phase that remains trapped at the interface.

The models including dynamic effects in the phase pressure difference lead to so-called pseudo-parabolic problems. For such models, but posed in homogeneous domains, existence and uniqueness of weak solutions are obtained in Cao and Pop (2015), Fan and Pop (2011) and Mikelić (2010). The case of vanishing capillary effects and the connection to hyperbolic conservation laws is studied in Duijn et al. (2007, 2013). For dynamic capillarity models in the heterogeneous case, but in the absence of an entry pressure, numerical schemes are discussed in Helmig et al. (2007). This situation is similar to the case analyzed in Cuesta and Pop (2009), where the interface is replaced by a discontinuity in the initial conditions. Also, variational inequality approaches have been considered in Helmig et al. (2009) for situations including an entry pressure. However, the conditions are simply postulated and no derivation is presented. 
In Sect. 2, we present the mathematical model. For simplicity, we consider the case when only the absolute permeability is different in the two blocks, all other parameters being the same. In Sect. 3, we derive the coupling conditions at the interface. These are the flux continuity and an extended pressure continuity. In the last section, we discuss different numerical approaches, and present numerical experiments that confirm the analysis in Sect. 3.

\section{Mathematical Model}

We consider the flow of two immiscible and incompressible phases in a one-dimensional heterogeneous porous medium. Letting $s_{w}$ denote the saturation of the wetting fluid, and $s_{n}$ the saturation of the non-wetting fluid, one has $0 \leq s_{w}, s_{n} \leq 1$. The porous medium is assumed to be saturated by the two fluids,

$$
s_{w}+s_{n}=1 .
$$

Mass balance holds for each phase (see Nordbotten and Celia 2012; Helmig 1997),

$$
\phi \frac{\partial s_{\alpha}}{\partial t}+\frac{\partial q_{\alpha}}{\partial x}=0, \quad \alpha=w, n
$$

where $\phi$ is the porosity assumed constant, and $q_{\alpha}$ denotes the volumetric velocity of the phase $\alpha$. These velocities satisfy the Darcy law

$$
q_{\alpha}=-\bar{k}(x) \frac{k_{r \alpha}\left(s_{\alpha}\right)}{\mu_{\alpha}} \frac{\partial p_{\alpha}}{\partial x}, \quad \alpha=w, n,
$$

where $\bar{k}(x)$ is the absolute permeability of the porous medium, $p_{\alpha}$ the pressure, $\mu_{\alpha}$ the viscosity and $k_{r \alpha}$ the relative permeability of the $\alpha$ phase. The functions $k_{r \alpha}$ are assumed known. Gravity effects are disregarded, as they have no influence on the interface conditions derived here. Substituting (3) into (2) gives

$$
\phi \frac{\partial s_{\alpha}}{\partial t}-\frac{\partial}{\partial x}\left(\bar{k}(x) \frac{k_{r \alpha}}{\mu_{\alpha}} \frac{\partial p_{\alpha}}{\partial x}\right)=0, \quad \alpha=w, n .
$$

In standard models, the phase pressure difference depends on the saturation, which is determined experimentally. An example in this sense is the Leverett relationship

$$
p_{n}-p_{w}=p_{c}\left(x, s_{w}\right)=\sigma \sqrt{\frac{\phi}{\bar{k}(x)}} J\left(s_{w}\right),
$$

where $\sigma$ is the interfacial tension, and $J$ a decreasing function.

The relationship in (5) is determined by measurements carried out under equilibrium condition. In other words, before measuring the pressure and saturation in a representative elementary volume, fluids have reached equilibrium and are at rest. However, processes of interest may not satisfy this condition, and dynamic effects have to be included. Alternatively to (5), in Hassanizadeh and Gray (1993) the following model is proposed

$$
p_{n}-p_{w}=p_{c}\left(x, s_{w}\right)-\bar{\tau} \frac{\partial s_{w}}{\partial t},
$$

The damping factor $\bar{\tau}$ is assumed to be known and constant. Summing the two equations in (4) and using (1), one gets

$$
\frac{\partial \bar{q}}{\partial x}=0
$$


where $\bar{q}=-\frac{\bar{k}(x) k_{r w}}{\mu_{w}} \frac{\partial p_{w}}{\partial x}-\frac{\bar{k}(x) k_{r n}}{\mu_{n}} \frac{\partial p_{n}}{\partial x}$ denotes the total flow. In the one-dimensional case, this means that $\tilde{q}$ is constant in space. Here, we assume it is constant in time as well, and is positive. This allows reducing the two-phase flow model to a scalar equation in terms of, say $s=s_{w}$. After rescaling the space $x$ with $L$, the time $t$ with $T$, and using the reference value $K$ for the absolute permeability and $\sigma \sqrt{\frac{\phi}{K}}$ for the pressure, one obtains

$$
\frac{\partial s}{\partial t}+\frac{\partial F}{\partial x}=0
$$

where the $F$ denotes the dimensionless flux of the wetting phase

$$
F=q f_{w}(s)+N_{c} k(x) \bar{\lambda}(s) \frac{\partial}{\partial x}\left(\frac{J(s)}{\sqrt{k(x)}}-N_{c} \tau \frac{\partial s}{\partial t}\right) .
$$

Here $q=\frac{\bar{q} T}{\phi L}>0$, and $k(x)=\frac{\bar{k}(x)}{K}$. Further, $f_{w}$ is the fractional flow function of the wetting phase,

$$
f_{w}=\frac{k_{r w}(s)}{k_{r w}(s)+k_{r n}(s) / M},
$$

with the mobility ratio $M=\mu_{n} / \mu_{w}$, the capillary number $N_{c}=\frac{\sigma \sqrt{\phi K}}{\mu_{n} q L}$, the dimensionless damping factor $\tau=\bar{\tau} \mu_{n}\left(\frac{\bar{q}}{\sigma \phi}\right)^{2}$ and $\bar{\lambda}(s)=k_{r n}(s) f_{w}(s)$. For simplicity, we assume here $N_{c}=1$, as different values of $N_{c}$ would not have any influence on the conditions derived below.

Throughout this work, we make the following assumptions

(A1) $k_{r w}, k_{r n}:[0,1] \rightarrow[0,1]$ are continuous differentiable functions satisfying

a) $k_{r w}$ is strictly increasing such that $k_{r w}(0)=0$ and $k_{r w}(1)=1$;

b) $k_{r n}$ is strictly decreasing such that $k_{r n}(0)=1$ and $k_{r n}(1)=0$;

(A2) $J$ is $(0,1] \rightarrow \mathbb{R}$ is continuous differentiable, decreasing which satisfies $J^{\prime}<0$ on $(0,1], J(1) \geq 0$ and $\lim _{s \searrow 0} J(s)=+\infty$

We consider a simple heterogeneous situation, where two adjacent homogeneous blocks are separated by an interface located at $x=0$. For the ease of presentation, we assume that all parameters and functional dependencies except the absolute permeability are the same. For the latter, we have

$$
k(x)=\left\{\begin{array}{l}
k^{-}, \text {if } x<0 \text { (the coarse medium) } \\
k^{+}, \text {if } x>0 \text { (the fine medium) }
\end{array}\right.
$$

Here $k^{-}>k^{+}>0$ are given.

Throughout this paper, the non-wetting phase may be oil, or $\mathrm{CO}_{2}$ or any other phase with non-wetting phase properties. For simplicity, below, we use as oil non-wetting phase. Also, by pressure we actually mean the phase pressure difference.

\section{Conditions at the Interface}

The model (8)-(9) is a parabolic equation, where the factor $\frac{1}{\sqrt{k}}$ appears under a second order spatial derivative. Since $k$ has a jump discontinuity at the interface, the model is only valid in each of the two blocks, and coupling conditions at $x=0$ have to be derived. Commonly, the 
Fig. 1 The function $h_{\epsilon}$

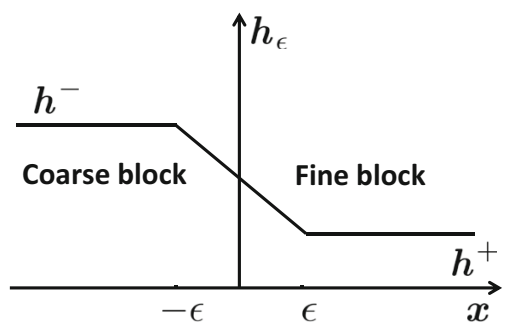

pressure continuity is taken as a second condition. However, this is shown to be inappropriate for entry-pressure models in the absence of dynamic effects $(\tau=0)$. This statement is made rigorous in Duijn et al. (1995) by regularizing $k$. Specifically, the interface is replaced by a thin layer in which $k$ decays continuously from $k^{-}$to $k^{+}$. Next to $k$, we will use the quantity

$$
h(x)=\sqrt{k(x)} .
$$

Clearly,

$$
h(x)=\left\{\begin{array}{l}
h^{-}=\sqrt{k^{-}}, \text {if } x<0, \\
h^{+}=\sqrt{k^{+}}, \text {if } x>0,
\end{array}\right.
$$

and $h^{-}>h^{+}>0$.

For given $\epsilon>0$, we approximate the interface $x=0$ by the interval $[-\epsilon, \epsilon]$ (the thin layer) and $h$ by a smooth function $h_{\epsilon}$, such that $h_{\epsilon}$ is monotonic in the small interval $[-\epsilon, \epsilon]$. Specifically, the discontinuous function $h$ is now approximated by the smooth function $h_{\epsilon}$, such that

$$
h_{\epsilon}(x)= \begin{cases}h^{-}, & \text {for } x<-\epsilon, \\ \hat{h}\left(\frac{x}{\epsilon}\right), & \text { for }-\epsilon<x<\epsilon, \\ h^{+}, & \text {for } x>\epsilon\end{cases}
$$

The function $\hat{h}$ is smooth and monotonic on $[-\epsilon, \epsilon]$ (see Fig. 1). With the given $\epsilon$, the solution corresponding to the regularized problem is denoted by $s_{\epsilon}$, the corresponding flux by $F_{\epsilon}$. In the expression for the flux, we replace $h$ by $h_{\epsilon}$. By taking $y=\frac{x}{\epsilon}$, we rescale $[-\epsilon, \epsilon]$ to $[-1,1]$. We define $v_{\epsilon}(y, t)=v_{\epsilon}\left(\frac{x}{\epsilon}, t\right)=s_{\epsilon}(x, t)$, and investigate its behavior when $\epsilon \searrow 0$. First, for $x \in[-\epsilon, \epsilon]$ (and thus $y \in[-1,1]$ ), from (8) one gets

$$
\frac{\partial}{\partial t} v_{\epsilon}(y, t)+\frac{1}{\epsilon} \frac{\partial}{\partial y} F_{\epsilon}(y, t)=0 .
$$

Assuming $\frac{\partial v_{\epsilon}}{\partial t}$ bounded uniformly with respect to $\epsilon$, passing $\epsilon$ to 0 gives $\lim _{\epsilon \searrow 0} \frac{\partial F_{\epsilon}}{\partial y}(y, t)=0$, implying

$$
\lim _{\epsilon \searrow 0} F_{\epsilon}(-1, t)=\lim _{\epsilon \searrow 0} F_{\epsilon}(1, t)
$$

In fact, this is nothing but the flux continuity at the interface, which is as expected.

For the second condition, we consider again $y \in[-1,1]$. From (9), one has

$$
F_{\epsilon}-q f_{w}\left(v_{\epsilon}\right)=h_{\epsilon}^{2} \bar{\lambda}\left(v_{\epsilon}\right) \frac{1}{\epsilon} \frac{\partial}{\partial y}\left(\frac{J\left(v_{\epsilon}\right)}{h_{\epsilon}}-\tau \frac{\partial v_{\epsilon}}{\partial t}\right) .
$$


Fig. 2 The strip $\Omega$

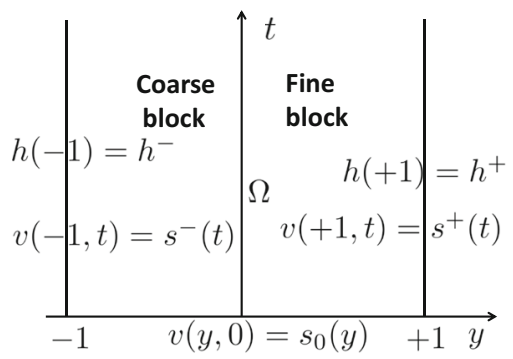

As before, let now $\epsilon \searrow 0$, assume that $v_{\epsilon}(y, t) \rightarrow v(y, t)$ and that during the limit process, the flux $F_{\epsilon}$ is bounded uniformly in $\epsilon$. Then, this gives

$$
\bar{\lambda}(v) \frac{\partial}{\partial y}\left(\frac{J(v)}{h(y)}-\tau \frac{\partial v}{\partial t}\right)=0, \quad \text { in } \Omega,
$$

where $\Omega$ denotes the strip $\Omega=\{(y, t):-1<y<1, t>0\}$ (see Fig. 2).

Along the boundary of $\Omega$, we define

$$
\begin{aligned}
& s^{-}(t):=s\left(0^{-}, t\right)=v(-1, t), \quad \text { for } t>0 . \\
& s^{+}(t):=s\left(0^{+}, t\right)=v(1, t),
\end{aligned}
$$

The goal is to understand how $s^{-}(t)$ and $s^{+}(t)$ are related, as well as $p^{-}(t)=\frac{J\left(s^{-}(t)\right)}{h^{-}}-$ $\tau \frac{\partial s^{-}(t)}{\partial t}, p^{+}(t)=\frac{J\left(s^{+}(t)\right)}{h^{+}}-\tau \frac{\partial s^{+}(t)}{\partial t}$. These are nothing but the left and right saturation and pressure at the interface. Note that (11) is an ordinary differential equation in $t$, where $y$ can be seen as a parameter. To define an initial condition, we let $s_{0}(y)$ be a smooth function $s_{0}:[-1,1] \rightarrow \mathbb{R}$ satisfying $s_{0}(-1)=s^{-}(0)$ and $s_{0}(+1)=s^{+}(0)$. Clearly, the choice of $s_{0}$ is not unique. Below we investigate the relation between $s^{-}(t)$ and $s^{+}(t)$, and its dependence of the regularization of $h$. Since $\bar{\lambda}(v)>0$, for $0<v<1$, and $\bar{\lambda}(0)=\bar{\lambda}(1)=0$, from (11), one has

$$
v=0, \quad \text { or } v=1,
$$

or if $0<v<1$,

$$
\frac{\partial}{\partial y}\left(\frac{J(v)}{h(y)}-\tau \frac{\partial v}{\partial t}\right)=0 .
$$

In other words

- $\frac{J(v)}{h(y)}-\tau \frac{\partial v}{\partial t}$ is constant in $y$, whenever $0<v<1$;

- $v(\cdot, t)$ is continuous with respect to $y$, for $y[-1,1]$;

- $v(y, \cdot)$ is $C^{1}$ with respect to $t$, for $t \geq 0$.

For the sake of understanding, we consider some particular cases.

\subsection{Constant Saturation at the Coarse Side of the Interface}

We let $s^{-} \in(0,1)$ and assume $s^{-}(t)=s^{-}$for all $t$. Let $s_{0}:[-1,1] \longrightarrow(0,1)$ be the given initial value, not necessarily compatible to $s^{-}: s_{0}(-1) \neq s^{-}$in general. We construct a solution for which the set $\Omega_{c}=\{(y, t) \in \Omega, 0<v(y, t)<1\}$ is connected. From (12), if $(y, t) \in \Omega, v$ solves the autonomous initial value problem: 
Fig. 3 The functions $\frac{J(v)}{h(y)}$ for various $y \in[-1,1]$ for $s^{-}>s^{*}$

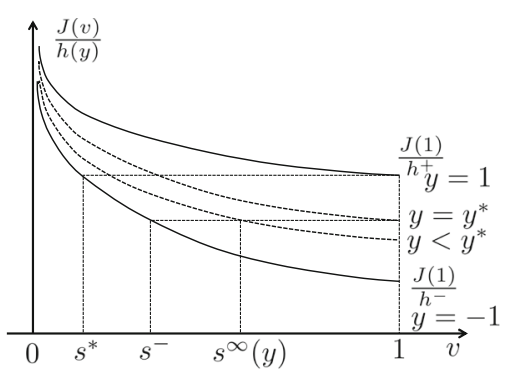

$$
\left(P_{y}\right)\left\{\begin{array}{l}
\tau \frac{\partial v}{\partial t}=\frac{1}{h(y)}\left(J(v)-\frac{h(y)}{h^{-}} J\left(s^{-}\right)\right), \quad \text { for } t>0, \\
v(y, 0)=s_{0}(y) .
\end{array}\right.
$$

By (A2) and the assumption on $h,\left(P_{y}\right)$ has a unique solution locally. Let $s^{*}$ be defined by

$$
\frac{J\left(s^{*}\right)}{h^{-}}=\frac{J(1)}{h^{+}} .
$$

We consider the cases $s^{-}>s^{*}$ and $s^{-} \leq s^{*}$, separately.

- Case 1: $s^{-}>s^{*}$

Note that

$$
\frac{J\left(s^{-}\right)}{h^{-}}<\frac{J\left(s^{*}\right)}{h^{-}}=\frac{J(1)}{h^{+}} .
$$

Since $h$ is decreasing function in $y$, there exists a unique $y^{*} \in(-1,1)$ such that

$$
\frac{J\left(s^{-}\right)}{h^{-}}=\frac{J(1)}{h\left(y^{*}\right)}<\frac{J(1)}{h^{+}} .
$$

We study now the long time behavior of $v(y, t)$. We distinguish the following sub-cases.

a) For $y \in\left(-1, y^{*}\right]$, define $s^{\infty}(y)$ as the unique solution of

$$
\frac{J\left(s^{\infty}(y)\right)}{h(y)}=\frac{J\left(s^{-}\right)}{h^{-}} .
$$

Clearly, $s^{\infty}(y)$ is the equilibrium point for $\left(P_{y}\right)$ and satisfies $s^{\infty}>s^{-}$(see Fig. 3).

Further, standard phase plane arguments show that, regardless of $s_{0}(y) \in(0,1), \lim _{t \rightarrow \infty} v(y, t)$ $=s^{\infty}(y)$. If $s_{0}(y)>s^{\infty}(y)$, the solution $v(y, t)$ decreases continuously from $v(y, 0)=s_{0}(y)$ to $v(y, \infty)=s^{\infty}(y)$. If $s_{0}(y)<s^{\infty}(y)$, the solution $v(y, t)$ increases continuously from $v(y, 0)=s_{0}(y)$ to $v(y, \infty)=s^{\infty}(y)$. Note that $s^{\infty}(-1)=s^{-}$, and that $s^{\infty}(y)$ is strictly increasing in $y$ up to $s^{\infty}\left(y^{*}\right)=1$. For $y>y^{*}$, we have $s^{\infty}(y)=1$.

b) For $y>y^{*}$, one has

$$
\tau \frac{\partial v}{\partial t}=\frac{J(v)}{h(y)}-\frac{J\left(s^{-}\right)}{h^{-}} \geq \frac{J(1)}{h(y)}-\frac{J\left(s^{-}\right)}{h^{-}}>0 .
$$


Fig. 4 The solution for $s^{-}>s^{*}$

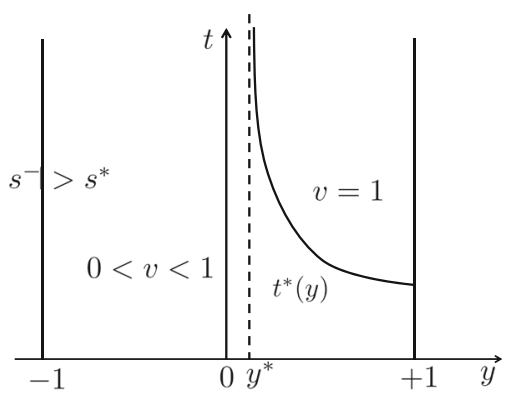

Fig. 5 Behavior of $s^{+}(t)$ for $t>0: s^{+}$is increasing to 1 for $t<t^{*}(1)$, and $s^{+}(t)=1$ for $t \geq t^{*}(1)$

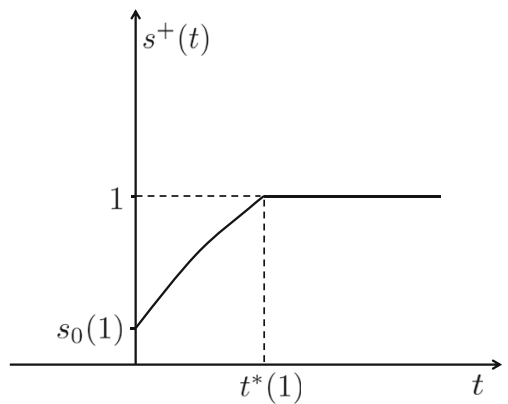

This implies the solution $v(y, t)$ increases in $t$ and reaches $v=1$ in finite positive time (see Fig. 4)

$$
0<t^{*}(y)<\frac{\tau\left(1-s_{0}(y)\right)}{\frac{J(1)}{h(y)}-\frac{J\left(s^{-}\right)}{h^{-}}} .
$$

Note: if $s_{0}(y)$ is non-decreasing, then $t^{*}(y)$ is decreasing to $t^{*}(1)>0$. The long time behavior of $v$ is summarized in

Proposition 1 Assume $s_{0}(y) \in(0,1)$ for all $y \in[-1,1]$ and let $s^{-}>s^{*}$, where $s^{*}$ is defined in (14). With $y^{*}$ in (15), one has:

a) if $y \in\left[-1, y^{*}\right)$ then $\lim _{t \rightarrow \infty} v(y, t)=s^{\infty}(y)$, where $s^{\infty}(y) \in(0,1]$ is given by (16).

Further, $s^{\infty}(\cdot)$ is strictly increasing from $s^{-}=s^{\infty}(-1)$ to $1=s^{\infty}\left(y^{*}\right)$;

b) if $y \in\left(y^{*}, 1\right]$, then there exists $t^{*}(y)>0$ such that $v(y, \cdot)$ is increasing in $t$ for all $t<t^{*}<\infty$, and $v(y, t)=1$ for all $t \geq t^{*}(y)$. Moreover, if $s_{0}(\cdot)$ is non-decreasing, then $t^{*}(\cdot)$ is decreasing to $t^{*}(1)>0$.

Corollary 1 In particular, at $y=1$, we have for $s^{+}(t)=v(1, t) . s^{+}(t)$ increases to 1 for $t \in\left(0, t^{*}(1)\right)$, where $0<t^{*}(1)<\tau\left(1-s_{0}(y)\right) /\left(\frac{J(1)}{h^{+}}-\frac{J\left(s^{-}\right)}{h^{-}}\right), s^{+}(t)=1$ for $t \geq t^{*}(1)$, as presented in Fig. 5.

This allows constructing an extended pressure condition, similar to Duijn et al. (1995). Consider the pressure

$$
p(y, t):=\frac{J(v(y, t))}{h(y)}-\tau \frac{\partial v}{\partial t},
$$

observe that

$$
p(-1, t)=p^{-}(t)=\frac{J\left(s^{-}\right)}{h^{-}}, \quad \text { and } \quad p(+1, t)=p^{+}(t)=\frac{J\left(s^{+}\right)}{h^{+}}-\tau \frac{\partial s^{+}}{\partial t} .
$$


With the entry pressure

$$
p_{e}^{+}:=\frac{J(1)}{h^{+}}
$$

since $s^{-}>s^{*}$, one has

$$
\frac{J\left(s^{-}\right)}{h^{-}}<p_{e}^{+}
$$

By Corollary 1, we obtain the condition:

$$
\begin{cases}p^{-}(t)=p^{+}(t), & \text { for } 0<t<t^{*}(1), \\ s^{+}(t)=1, & \text { for } t \geq t^{*}(1) .\end{cases}
$$

In other words, the pressure remains continuous for $t<t^{*}(1)$ and oil keeps flowing into the fine material although $p^{-}$is below the entry pressure. This effect is due to incorporating dynamic effects in the phase pressure difference and would not be possible in their absence $(\tau=0)$.

- Case 2: $s^{-}<s^{*}$

For any $y \in[-1,1]$, with $s^{\infty}(y)$ defined by (16), one has

$$
\frac{J\left(s^{\infty}(y)\right)}{h(y)}=\frac{J\left(s^{-}\right)}{h^{-}}>\frac{J\left(s^{*}\right)}{h^{-}}=\frac{J(1)}{h^{+}} .
$$

Therefore, there exists an $\tilde{s} \in\left(s^{-}, 1\right)$, such that

$$
\frac{J\left(s^{\infty}(y)\right)}{h(y)}=\frac{J\left(s^{-}\right)}{h^{-}}=\frac{J(\tilde{s})}{h^{+}},
$$

which, in view of the monotonicity of $h$ and $J$, gives $s^{\infty}<\tilde{s}<1$. Consequently, this case is similar to the sub-case $y<y^{*}$ before.

Proposition 2 Assume $s_{0}(y) \in(0,1)$ for all $y \in[-1,1]$ and let $s^{-}<s^{*}($ see $(14))$. Then,

$$
\lim _{t \rightarrow \infty} v(y, t)=s^{\infty}(y) \text { for all } y \in(-1,1),
$$

with $s^{\infty}(y)$ given by (16). In particular, for $y=1$, one can define $s_{+}^{\infty}=s^{\infty}(1)$ as the unique solution of

$$
\frac{J\left(s_{+}^{\infty}\right)}{h^{+}}=\frac{J\left(s^{-}\right)}{h^{-}} .
$$

Corollary 2 At $y=1$, we have for $s^{+}(t)=v(1, t)$ :

$$
\lim _{t \rightarrow \infty} s^{+}(t)=s_{+}^{\infty} \in(0,1) .
$$

In other words, the pressure remains continuous at the interface for all $t>0$ :

$$
p^{+}(t)=\frac{J\left(s^{+}(t)\right)}{h^{+}}-\tau \frac{\partial s^{+}}{\partial t}=\frac{J\left(s^{-}\right)}{h^{-}}=p^{-} .
$$

Note that unlike in (20), the pressure remains continuous for all $t>0$.

All results refer to the case $J(1)>0$, hence to the entry pressure model. If instead, $J(1)=$ 0 (no entry pressure), $s^{*}=1$ and the analysis before leads to $s^{-}<1$, and $p^{-}(t)=p^{+}(t)$ for all $t>0$ (see Helmig et al. 2007).

Another special case is when $\tau \searrow 0$. Then, the time $t^{*}(y)$ in Proposition 1 and Corollary 1 approaches to 0 , and if $s^{-}>s^{*}$ the pressure becomes discontinuous instantaneously. This is, in fact, exactly the behavior in Duijn et al. (1995) for equilibrium models. 


\subsection{Non-constant Saturation at the Coarse Side of the Interface}

The results before are obtained for a constant saturation at the coarse side of the interface. Here, we generalize these results. We let $p^{ \pm}(t)=\frac{J\left(s^{ \pm}(t)\right)}{h^{ \pm}}-\tau \frac{\partial s^{ \pm}(t)}{\partial t}$ be the phase pressure difference at the two sides of the interface and derive an extended pressure condition similar to (20) and (22). With the entry pressure $p_{e}^{+}$defined in (19), we assume $p^{-}(t)$ given, and distinguish the following cases.

- Case 1: $p^{-}(t)>p_{e}^{+}$for all $t>0$

Again, for $y \in(-1,1), v(y, t)$ solves

$$
\left(P_{y}\right)\left\{\begin{array}{l}
\frac{J(v(y, t))}{h(y)}-\tau \frac{\partial v}{\partial t}=p^{-}(t), \quad \text { for } t>0, \\
v(y, 0)=s_{0}(y) .
\end{array}\right.
$$

Assume $s_{0}(y) \in(0,1)$, then the equation holds in the neighborhood of $t=0$. We show that in this case, $v(y, t)<1$ for any $t$, and hence $p^{-}=p(y, t)$, for any $y \in(-1,1)$. Assume $v\left(y, t^{*}\right)=1$ for some $t^{*}<\infty$. For $t<t^{*}$ one has

$$
\tau \frac{\partial v}{\partial t}=\frac{1}{h(y)}\left(J(v(y, t))-h(y) p^{-}(t)\right) .
$$

By the monotonicity of $h$,

$$
h(y) p^{-}(t)>h^{+} p^{-}(t)>h^{+} p_{e}^{+}=J(1),
$$

implying that, at $t=t^{*}$, one has

$$
\tau \frac{\partial v}{\partial t}\left(t^{*}\right)=\frac{J(1)}{h(y)}-p^{-}(t)<0 .
$$

This shows that $v(y, \cdot)$ cannot grow to 1 for $t \nearrow t^{*}$. Therefore, no finite $t^{*}$ exists, such that $v\left(y, t^{*}\right)=1$, implying $v(y, t) \in(0,1)$ for all $t$. We have proved

Proposition 3 Assume $s_{0}(y) \in(0,1)$ for all $y \in[-1,1]$ and let $p^{-}(t)>p_{e}^{+}$for all $t>0$. Then, for all $y \in[-1,1]$ and $t>0$, one has $v(y, t) \in(0,1)$.

Corollary 3 At $y=1$, we get $s^{+}(t)=v(1, t) \in(0,1)$ for all $t>0$, and therefore, the pressure remains continuous at both sides of the interface for all $t>0$

$$
p^{-}(t)=p^{+}(t)
$$

As in Sect. 3.1, if the model involves no entry pressure $(J(1)=0)$, one has $p_{e}^{+}=0$. Then, $p^{-}(t) \geq p_{e}^{+}=0$, and the pressure is continuous for all $t>0$.

- Case 2: $0<p^{-}(t)<p_{e}^{+}$for all $t>0$

We assume first that an $\epsilon$ exists such that $0<p^{-}(t)<p_{e}^{+}-\epsilon$ for all $t>0$. Further, we assume that the initial condition $s_{0}:(-1,1) \rightarrow \mathbb{R}$ is non-decreasing and smooth, and satisfies $s_{0}(y) \in(0,1)$ for any $y$. We have

Proposition 4 Assume there exists $\epsilon>0$ such that $0<p^{-}(t)<p_{e}^{+}-\epsilon$ for all $t>0$, and that $s_{0}$ is non-decreasing and smooth. Then, there exists a $t^{*}>0$ such that

$$
\begin{cases}p^{-}(t)=p^{+}(t), & \text { for } 0<t<t^{*}, \\ s^{+}(t)=1, & \text { for } t \geq t^{*}\end{cases}
$$


Proof Clearly, if $v(y, t)<1$, then $v(y, \cdot)$ solves

$$
\left(P_{y}\right)\left\{\begin{array}{l}
\frac{J(v(y, t))}{h(y)}-\tau \frac{\partial v}{\partial t}=p^{-}(t), \quad \text { for } t>0 \\
v(y, 0)=s_{0}(y)
\end{array}\right.
$$

First we prove the monotonicity of $v(\cdot, t)$. Specifically, for all $t$ such that $v(\cdot, t)<1$ uniformly in $y$, one also has $v(\cdot, t)$ is non-decreasing. To see this, we differentiate (26) with respect to $y$ to obtain

$$
\tau \frac{\partial^{2} v}{\partial t \partial y}-\frac{J^{\prime}(v)}{h(y)} \frac{\partial v}{\partial y}=-\frac{h^{\prime}}{h^{2}} J(v)
$$

For a fixed $y$, this has the general form:

$$
\tau \dot{u}=f u+b,
$$

with $u=\frac{\partial v}{\partial y}, f=\frac{J^{\prime}(v)}{h(y)}<0, b=-\frac{h^{\prime}}{h^{2}} J(v)$. Clearly, $u(0)=s_{0}^{\prime} \geq 0$. Assuming that a $\bar{t}>0$ exists such that $u(\bar{t})=0$ and $u(t)>0$ for any $t \in(0, \bar{t}]$, one gets:

$$
\dot{u}(\bar{t})=f(\bar{t}) u(\bar{t})+b(\bar{t})=b(\bar{t})>0 .
$$

On the other hand, one has

$$
\dot{u}(\bar{t})=\lim _{\Delta t \searrow 0} \frac{u(\bar{t})-u(\bar{t}-\Delta t)}{\Delta t} \leq 0,
$$

which contradicts the above.

Now, we proceed by proving the conclusion of the proposition. Since $p^{-}(t)<p_{e}^{+}-\epsilon$, by the continuity of $h$, there exists $\delta>0$ such that

$$
\frac{h(y)}{h^{+}} p^{-}(t)<p_{e}^{+}-\epsilon / 2, \text { for } 1-\delta<y<1 .
$$

Hence, for $y \in(1-\delta, 1)$ and $t>0$, we have

$$
J(v)-h(y) p^{-}(t)>J(v)-h^{+} p_{e}^{+}+\epsilon h^{+} / 2=J(v)-J(1)+\epsilon h^{+} / 2 .
$$

This implies

$$
\tau h(y) \frac{\partial v}{\partial t}>J(v)-J(1)+\epsilon h^{+} / 2>\epsilon h^{+} / 2 .
$$

Clearly, a finite $t=t(y)>0$ exists such that $v(y, t(y))=1$ and $v(y, t)$ for $t<t(y)$. By the monotonicity of $v$ and $s_{0}$, taking $t^{*}=t(1)$, one has $v(y, t)<1$ for any $y \in[-1,1]$ and $t<t^{*}$. Therefore, $v(y, t)$ solves $\left(P_{y}\right)$ for all $t<t^{*}$ and all $y$, hence $p(y, t)$ remains continuous, in particular, $p^{-}(t)=p^{+}(t)$.

Observe that, for fixed $y \in(-1,1), v(y, t)$ solving $\left(P_{y}\right)$ and $w(y, t)$ solving

$$
\left(P_{y}^{\prime}\right)\left\{\begin{array}{l}
\tau h(y) \frac{\partial w}{\partial t}=J(w)-h(y)\left(p_{e}^{+}-\epsilon\right), \quad t>0, \\
w(y, 0)=s_{0}(y)
\end{array}\right.
$$

are ordered. Specifically, as long as both $v$ and $w$ remain less than 1 , one has $w(y, t) \leq v(y, t)$. To see this, we let $u=v-w$ and define $u_{-}=\min \{0, u\}$. Then, subtracting the equations in $\left(P_{y}\right)$ and $\left(P_{y}^{\prime}\right)$, and multiplying the result by $u_{-}$gives

$$
\frac{h(y)}{2} \frac{\partial}{\partial t}\left(u_{-}\right)^{2}=(J(v)-J(w)) u_{-}-h(y)\left(p^{-}(t)-\left(p_{e}^{+}-\epsilon\right)\right) u_{-} \leq 0,
$$


Fig. 6 The function $t(y)$

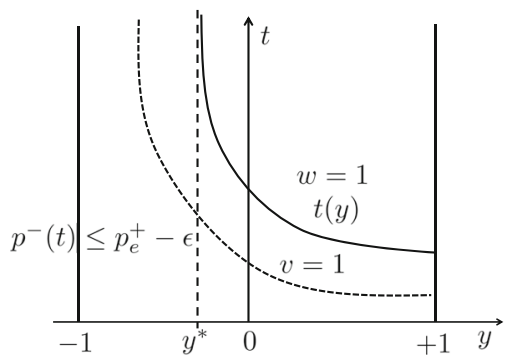

Here we have used the monotonicity of $J$. Since at $t=0$ one has $u_{-}(y, 0)=0$, integrating in time gives $u_{-}(y, t)=0$, implying the ordering. Hence $w$ is a lower bound for $v$, and therefore analyzing $w$ makes sense. Let $y^{*} \in(-1,1)$ be defined by

$$
h\left(y^{*}\right)=\frac{J(1)}{p_{e}^{+}-\epsilon / 2}=h^{+} \frac{J(1)}{J(1)-\epsilon / 2 h^{+}} .
$$

Then for $y>y^{*}$, a $\delta>0$ exists such that $h(y)=h\left(y^{*}\right)-\delta<h\left(y^{*}\right)$, as long as $w(y, t)<1$, one has

$$
\begin{aligned}
\tau \frac{\partial w}{\partial t}(y, t) & =\frac{J(w)}{h(y)}-\left(p_{e}^{+}-\epsilon\right) \geq \frac{J(1)}{h\left(y^{*}\right)}-\left(p^{+}-\epsilon\right)+\left(\frac{1}{h(y)}-\frac{1}{h\left(y^{*}\right)}\right) J(1) \\
& =\frac{\delta}{h(y) h\left(y^{*}\right)} J(1)>0 .
\end{aligned}
$$

This shows that $w$ reaches the cutoff value $w=1$ at finite time $t=t(y)$. If the function $s_{0}(y)$ is constant, it is straightforward to show that $t(y)$ is strictly decreasing to $t(1)>0$ (see Fig. 6). At $y=y^{*}, w=1$ satisfies the equation. Therefore, $w \equiv 1$ is an equilibrium solution, implying that $w\left(y^{*}, t\right)$ solving $\left(P_{y}^{\prime}\right)$ satisfies $w\left(y^{*}, t\right)<1$ for all $t$, and thus

$$
\lim _{y \searrow y^{*}} t(y)=\infty \text {. }
$$

Remark 1 In fact, the argument above is an alternative proof for Proposition 4. Since $s^{+}(t)=$ $v(1, t)>w(1, t)$, and $w(1, t(1))=1$ for $t(1)<\infty,(25)$ follows immediately.

Based on the analysis before, we discuss particular examples where oil trapping may occur. Defining $p(y, t):=\frac{J(v)}{h(y)}-\tau \frac{\partial v}{\partial t}$, in the transition region (the blown up interface) $\Omega=\{(y, t)$ : $-1<y<1, t>0\}$, we have

$$
\left\{\begin{array}{l}
\bar{\lambda}(v) \frac{\partial}{\partial y}\left(\frac{J(v)}{h(y)}-\tau \frac{\partial v}{\partial t}\right)=0, \\
p(t)=p^{-}(t), \quad t>0, \\
v(y, 0)=s_{0}(y), \quad-1<y<1,
\end{array}\right.
$$

which implies that either $v=0$ or $v=1$, or

$$
h(y) \tau \frac{\partial v}{\partial t}=J(v)-h(y) p^{-}(t) .
$$

To understand the trapping, we now take $s_{0}(y)=1$, for $-1<y<1$, and give the pressure at the coarse side of the interface for $t>0$. We assume the following behavior (see Fig. 7):

a) There exists $T_{1}>0$ such that $0<p^{-}(t)<p_{e}^{-}$for $t \in\left(0, T_{1}\right)$, where $p_{e}^{-}=\frac{J(1)}{h^{-}}$. 
Fig. 7 The function $\left.p^{-(} t\right)$

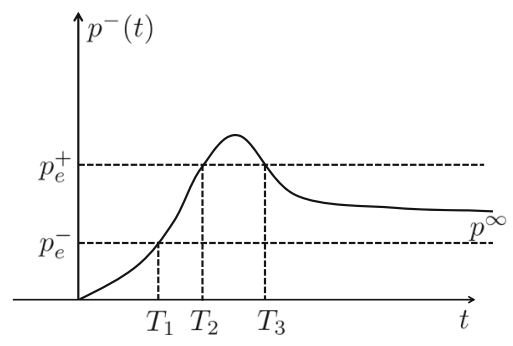

In this case, we have

$$
J(1)-h(y) p^{-}(t)=h^{-} p_{e}^{-}-h(y) p^{-}(t)>0 .
$$

Let $y \in[-1,1]$ be fixed. Assume that a finite $\tilde{t}>0$ and $\delta>0$ exist such that $v(y, t)=1$ for all $t<\tilde{t}$ and $v(y, \tilde{t})<1$ for $t \in(\tilde{t}, \tilde{t}+\delta)$, then one has $\frac{\partial v}{\partial t}(y, \tilde{t}) \leq 0$. Whereas by assumption for $t \in(\tilde{t}, \tilde{t}+\delta)$, one has

$$
\frac{\partial v}{\partial t}(y, \tilde{t})=\frac{1}{\tau}\left(\frac{J(1)}{h(y)}-p^{-}(\tilde{t})\right)>0,
$$

This gives a contradiction.

b) There exists a $T_{2}>T_{1}$ such that $p_{e}^{-}<p^{-}(t)<p_{e}^{+}$, for $t \in\left(T_{1}, T_{2}\right)$.

Since $p_{e}^{-}<p^{-}(t)<p_{e}^{+}$, one has

$$
\frac{J(1)}{h^{-}}<p^{-}(t)<\frac{J(1)}{h^{+}},
$$

For $T_{1}<t<T_{2}$, we define $\tilde{y}(t)$ by

$$
h(\tilde{y}(t)):=\frac{J(1)}{p^{-}(t)} .
$$

Note that the definition makes sense, as $p_{e}^{-}<p^{-}(t)<p_{e}^{+}$implies $\frac{1}{h^{-}}<\frac{p^{-}(t)}{J(1)}<\frac{1}{h^{+}}$, and $h(\cdot)$ is a monotone, continuous interpolation between $h^{+}$and $h^{-}$. Since $p^{-}$is increasing in $\left[T_{1}, T_{2}\right]$, this implies that $\tilde{y}(\cdot)$ is increasing and $\tilde{y}\left(T_{1}\right)=-1, \tilde{y}\left(T_{2}\right)=1$. Then, for $y>\tilde{y}(t)$, we have

$$
J(1)-h(y) p^{-}(t)>J(1)-h(\tilde{y}(t)) p^{-}(t)=0 .
$$

Similarly, for $y<\tilde{y}(t)$, we have

$$
J(1)-h(y) p^{-}(t)<0 .
$$

Furthermore, since $v\left(y, T_{1}\right)=1$ for all $y \in(-1,1)$, from (28), (29), one get for all $t \in$ $\left(T_{1}, T_{2}\right)$

$$
v(y, t)=1, \quad \text { for } y>\tilde{y}(t) \text {, and } v(y, t)<1 \text {, for } y<\tilde{y}(t) .
$$

Thus $y=\tilde{y}(t)$ defines a free boundary in the transition region, separating regions where $v=1$ from regions where $v<1$.

Remark 2 In this context, for $t<T_{2}$ one has $s^{+}(t)=v(1, t)=1$, and oil remains trapped in the coarse medium at the interface.

c) There exists $T_{3}>T_{2}$ such that $p^{-}>p_{e}^{+}$for $t \in\left(T_{2}, T_{3}\right)$. 
Based on the above analysis, we have $v\left(y, T_{2}\right)<1$ for all $y \leq 1$, since $\tilde{y}\left(T_{2}\right)=1$. Further, one also has

$$
J(1)-h(y) p^{-}(t)<J(1)-h(y) p_{e}^{+}<J(1)-h^{+} p_{e}^{+}=0 .
$$

As before, one cannot obtain $v(y, \tilde{t})=1$ for some $\tilde{t}>T_{2}$, since at $\tilde{t}$, it holds

$$
\frac{\partial v}{\partial t}(y, \tilde{t})=\frac{1}{\tau}\left(\frac{J(1)}{h(y)}-p^{-}(\tilde{t})\right)<0 .
$$

Therefore, $v(y, t)<1$ for $-1<y<1$ and $T_{2}<t<T_{3}$. Consequently, we have

$$
p^{-}(t)=p^{+}(t) \text { for } T_{2}<t<T_{3} \text {. }
$$

Remark 3 Next to the pressure continuity, this shows that oil starts flowing into the fine region for $t>T_{2}$.

d) For all $t>T_{3}, p^{-}<p_{e}^{+}$.

We assume $p^{-}(\cdot)$ decreasing and $\lim _{t \rightarrow \infty} p^{-}(t)=p^{\infty} \in\left(p_{e}^{-}, p_{e}^{+}\right)$. Given $y \in[-1,1]$, we compare the solution $v(y, t)$ and $w(y, t)$ of

$$
\begin{aligned}
& h(y) \tau \frac{\partial v}{\partial t}=J(v)-h(y) p^{-}(t), \\
& h(y) \tau \frac{\partial w}{\partial t}=J(w)-h(y) p^{\infty},
\end{aligned}
$$

for all $t>T_{3}$, with $v\left(y, T_{3}\right)=w\left(y, T_{3}\right)<1$.

Since $J$ is decreasing and $p^{-}(t)>p^{\infty}$, one gets $v(y, t) \leq w(y, t)$ for $-1<y<1, t>$ $T_{3}$. Further, there exists $y^{*} \in(-1,1)$ such that

$$
h\left(y^{*}\right)=\frac{J(1)}{p^{\infty}} \in\left(h^{+}, h^{-}\right) .
$$

This gives for $y>y^{*}$

$$
J(1)-h(y) p^{\infty}>0 .
$$

As before, there exists $t(y)<\infty$, such that $w(y, t)=1$ for $t>t(y)$.

Similarly, for $y<y^{*}$, one has

$$
J(1)-h(y) p^{\infty}<0,
$$

implying $w(y, t)<1$ for all $t>T_{3}$. In this case,

$$
\lim _{t \rightarrow \infty} w(y, t)=s^{\infty}(y),
$$

where $s^{\infty}(y)$ is defined by

$$
J\left(s^{\infty}(y)\right)=h(y) p^{\infty} .
$$

Observe that, since $h(y)>h\left(y^{*}\right), J\left(s^{\infty}(y)\right)=\frac{h(y)}{h\left(y^{*}\right)} J(1)>J(1)$, so $s^{\infty}(y) \in(0,1)$. For $y=-1$ one has

$$
J\left(s^{\infty}(-1)\right)=\frac{h^{-}}{h\left(y^{*}\right)} J(1)<\frac{h^{-}}{h^{+}} J(1)=J\left(s^{*}\right),
$$

giving $s^{\infty}(-1)>s^{*}$. Further, if $p^{\infty}=p_{e}^{+}=\frac{J(1)}{h^{+}}$, then $s^{\infty}(-1)=s^{*}$. 
Fig. 8 Saturation inside the thin layer approximating the interface

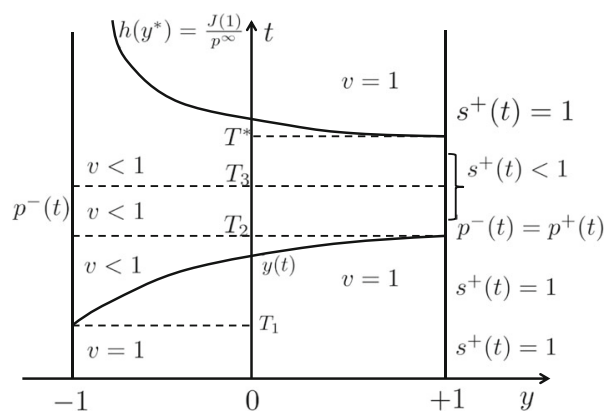

This analysis shows that, if $p^{-}(t)$ behaves as in Fig. 7, and $p^{\infty} \in\left(p_{e}^{-}, p_{e}^{+}\right)$, a $T^{*}<\infty$ exists such that $s^{+}(t)=1$, for $t>T^{*}$, and $p^{-}(t)=p^{+}(t)$ for $T_{3}<t<T^{*}$. This means up to $T^{*}$, oil flows into the fine material, while trapping occurs for $t>T^{*}$. This behavior is sketched in Fig. 8.

Remark 4 Compared to the equilibrium case $(\tau=0)$, a striking difference appears. If $\tau=0$ for a pressure $p^{-}(t)$ behaving as in Fig. 7, no oil flows into the fine layer for any $t>T_{3}$. If $\tau>0$, oil continues flowing for $t>T_{3}$ and up to a time $T^{*}<\infty$, the delay time. This delay appears, as discussed, if $\lim _{t \rightarrow \infty} p^{-}(t)=p^{\infty} \in\left(p_{e}^{-}, p_{e}^{+}\right)$.

The following result extends the statement in the remark to a more general situation.

Proposition 5 Let $p^{-}(t) \leq p_{e}^{+}$be such that $\int_{0}^{\infty}\left(p_{e}^{+}-p^{-}(t)\right) \mathrm{d} t>\tau$ and let $s_{0}(y) \in(0,1)$. Further, let $s^{+}$be the solution of

$$
\left\{\begin{array}{l}
h^{+} \tau \frac{\mathrm{d} s^{+}}{\mathrm{d} t}=J\left(s^{+}\right)-h^{+} p^{-}(t), \text { for } t>0, \\
s^{+}(0)=s_{0} .
\end{array}\right.
$$

Then there exists $T^{*}<\infty$ such that $s_{0}<s^{+}(t)<1$ for all $0<t<T^{*}$ and $s^{+}\left(T^{*}\right)=1$.

Proof Since $p^{-}(t) \leq p_{e}^{+}=\frac{J(1)}{h^{+}}$, and $J\left(s^{+}\right)$is strictly decreasing, we have

$$
J\left(s^{+}\right)-h^{+} p^{-}(t)>0, \quad \text { for } s^{+} \in(0,1) .
$$

Therefore, $s^{+}(t)$ is strictly increasing whenever $s^{+}<1$. Furthermore, we have

$$
h^{+} \tau \frac{d s^{+}}{d t}>h^{+}\left(p_{e}^{+}-p^{-}(t)\right)
$$

giving for all $t$ such that $s^{+}(t)<1$ and

$$
s^{+}(t)>s_{0}+\frac{1}{\tau} \int_{0}^{t}\left(p_{e}^{+}-p^{-}(\zeta)\right) \mathrm{d} \zeta .
$$

For convenience, define $f(t)=\frac{1}{\tau} \int_{0}^{t}\left(p_{e}^{+}-p^{-}(\zeta)\right) \mathrm{d} \zeta$. Clearly, $f(0)=0, \partial_{t} f \geq 0$ and $f(\infty)>1$. Hence, there exists $t^{*}<\infty$ for which $f\left(t^{*}\right)=1-s_{0}$. Consequently, there exists $T^{*}<t^{*}$ for which $s^{+}\left(T^{*}\right)=1$, and since $s^{+}$is increasing, one has $s_{0}<s^{+}(t)<1$ for all $t<T^{*}$. 
Remark 5 A lower bound for $s^{+}$is $w=w(t)$, the solution of

$$
\left\{\begin{array}{l}
h^{+} \tau \frac{d w}{d t}=J(w)-J(1), \quad \text { for } t>0, \\
w(0)=s_{0} .
\end{array}\right.
$$

Since $J:(0,1] \rightarrow \mathbb{R}^{+}$is locally Lipschitz, then for all $t>0, w(\cdot)$ is strictly increasing, $w(t)<1$, and $\lim _{t \rightarrow \infty} w(t)=1$. By a comparison argument, $s(t)>w(t)$ for all $t>0$.

\subsection{Comparison of Extended Pressure Conditions with Static Case}

In this section, we show the difference in the pressure conditions appearing in the equilibrium and non-equilibrium models between static case and dynamic case. As proved in Duijn et al. (1995), if $s^{-} \geq s^{*}$, then one has

$$
s^{+}=1,
$$

and no oil flows into the fine medium. Further, if $s^{-}<s^{*}$, then $s^{+}<1$, but the pressure continuous:

$$
[p]=p^{-}(t)-p^{+}(t)=0 .
$$

Then, oil flows into the fine medium.

Conversely, given $p^{-}(t)=\frac{J\left(s^{-}(t)\right)}{h^{-}}$, the pressure at the interface on the coarse side and assuming that

$$
p^{-}(t) \leq p_{e}^{+}=\frac{J(1)}{h^{+}},
$$

it implies

$$
J\left(s^{-}\right) \leq \frac{h^{-}}{h^{+}} J(1)=J\left(s^{*}\right),
$$

and therefore $s^{-} \geq s^{*}$. In this case, one also has $s^{+}=1$.

Similarly, $p^{-}(t)>p_{e}^{+}$implies $s^{-}<s^{*}$, and the pressure is continuous:

$$
p^{-}(t)=p^{+}(t) \text {. }
$$

Referring to Fig. 7, if $p^{-}(t) \geq p_{e}^{+}$for $t>T_{2}$, the matching conditions in static and dynamic case are the same. Specifically, $s^{+}=1$ for $t<T_{2}$, and $p^{-}(t)=p^{+}(t)$ for $t \in\left(T_{2}, T_{3}\right)$. Assuming now $p^{-}(t)$ is decreasing for $t>T_{3}$, with $p^{-}\left(T_{3}\right)=p_{e}^{+}$and $\lim _{t \rightarrow \infty} p^{-}(t)=p^{\infty} \in\left(p_{e}^{-}, p_{e}^{+}\right)$, we have (also see Fig. 8):

$s^{+}(t)=1$, for $t>T_{3}$ in the equilibrium case $\tau=0$,

$s^{+}(t)<1$, for $t \in\left(T_{3}, T^{*}\right)$, and $s^{+}(t)=1$ for $t \geq T^{*}$ in the non-equilibrium case.

In other words, a delay $\left(T^{*}-T_{3}\right)$ appears in the non-equilibrium case before trapping occurs.

In fact, the delay can be infinite. An extreme situation, when $s(t)<1$ for all $t>0$, can be constructed. To see this, we assume $J:(0,1] \rightarrow \mathbb{R}$ locally Lipschitz, and study the behavior of $s^{+}$solving

$$
\left\{\begin{array}{l}
h^{+} \tau \frac{d s^{+}}{d t}=J(s)-h^{+} p^{-}(t), \quad \text { for } t>0, \\
s(0)=s_{0} \in(0,1),
\end{array}\right.
$$

with appropriately chosen $p^{-}$satisfying $p^{-}(t)<p_{e}^{+}$for all $t>0$. 
First, note that an $L>0$ exists such that for all $s \in\left[s_{0}, 1\right]$

$$
0 \leq J(s)-J(1) \leq L(1-s) .
$$

Hence, an upper bound to $s^{+}$is the solution $u$ of

$$
\left\{\begin{array}{l}
\tau \frac{\mathrm{d} u}{\mathrm{~d} t}=\frac{L}{h^{+}}(1-u)+\left(p_{e}^{+}-p^{-}(t)\right), \text { for } t>0, \\
u(0)=s_{0}
\end{array}\right.
$$

Let now $v=1-u$. Then

$$
\tau \frac{\mathrm{d} v}{\mathrm{~d} t}+\frac{L}{h^{+}} v=-\left(p_{e}^{+}-p^{-}(t)\right), t>0 .
$$

This gives

$$
v(t)=\left(1-s_{0}\right) \mathrm{e}^{-\frac{L}{\tau h^{+}} t}-\frac{1}{\tau} \int_{0}^{t} \mathrm{e}^{\frac{L}{\tau h^{+}}(z-t)}\left(p_{e}^{+}-p^{-}(z)\right) \mathrm{d} z,
$$

and the upper bound for $s^{+}$reads

$$
u(t)=1-\left(1-s_{0}\right) \mathrm{e}^{-\frac{L}{\tau h^{+}} t}+\frac{1}{\tau} \int_{0}^{t} \mathrm{e}^{\frac{L}{\tau h^{+}}(z-t)}\left(p_{e}^{+}-p^{-}(z)\right) \mathrm{d} z .
$$

Thus, if $p^{-}(t)<p_{e}^{+}$is such that

$$
\int_{0}^{\infty} \mathrm{e}^{\frac{L}{\tau h^{+}} z}\left(p_{e}^{+}-p^{-}(z)\right) \mathrm{d} z \leq\left(1-s_{0}\right) \tau,
$$

we obtain $s^{+}(t)<1$ for all $t>0$, and consequently, the pressure remains continuous for all $t>0$, whereas oil flows into the fine layer.

\section{Numerical Method and Examples}

In this section, we provide some numerical examples to illustrate how the dynamic effects influence the flow of the oil across the interface between two homogeneous blocks. For simplicity, in (8)-(9), we take $\phi=1$. This gives

$$
\begin{gathered}
\frac{\partial s}{\partial t}+\frac{\partial F}{\partial x}=0 \\
F=q f_{w}(s)+h^{2} \bar{\lambda}(s) \frac{\partial}{\partial x}\left(\frac{J(s)}{h(x)}-\tau \frac{\partial s}{\partial t}\right),
\end{gathered}
$$

for $t>0$, and $x \in(-l, l)$. The boundary and initial conditions are given below.

\subsection{Linear Numerical Scheme}

For the discretization of (30) and (31), we decompose the interval $(-l, l)$ into $2 N+1$ cells $:-l=x_{-N-1 / 2}<x_{-N+1 / 2}<\cdots<x_{-1 / 2}<x_{1 / 2}<\cdots<x_{N-1 / 2}<x_{N+1 / 2}=l$, where the grid is uniform with $\Delta x=\frac{2 l}{2 N+1}$, we let $x_{j}=j \Delta x$ for $j \in\{-N-1 / 2, \ldots, N+1 / 2\}$. The discontinuity of $h(x)$ is at $x=0$. With $\Delta t>0$ a given time step, the fully discrete scheme is:

$$
\frac{s_{i-1 / 2}^{n}-s_{i-1 / 2}^{n-1}}{\Delta t}=-\frac{F_{i}^{n}-F_{i-1}^{n}}{\Delta x},
$$


where $s_{i-1 / 2}^{n-1}$ is the approximation of $s(x, t)$ at $x=x_{i-1 / 2}$ and at $t=t^{n}=n \Delta t$. Since $q>0$, if $i \neq 0$ the upwind flux $F_{i}^{n}$ at $x=i \Delta x$ and $t=t^{n}$ is defined as

$$
\begin{aligned}
F_{i}^{n}= & q f_{w}\left(s_{i-1 / 2}^{n-1}\right)+h^{+/-} \bar{\lambda}\left(\frac{s_{i-1 / 2}^{n-1}+s_{i+1 / 2}^{n-1}}{2}\right) \cdot\left(J^{\prime}\left(\frac{s_{i-1 / 2}^{n-1}+s_{i+1 / 2}^{n-1}}{2}\right)\right. \\
& \left.\cdot\left(\frac{s_{i+1 / 2}^{n}-s_{i-1 / 2}^{n}}{\Delta x}\right)-\frac{h^{+/-} \tau}{\Delta x}\left(\frac{s_{i+1 / 2}^{n}-s_{i+1 / 2}^{n-1}}{\Delta t}-\frac{s_{i-1 / 2}^{n}-s_{i-1 / 2}^{n-1}}{\Delta t}\right)\right) .
\end{aligned}
$$

Here $h^{+/-}$means $h^{-}$if $i<0$, or $h^{+}$if $i>0$.

At $i=0$, we introduce two saturation unknowns $s^{-, n}, s^{+, n}$ and define the $F^{-, n}$, and $F^{+, n}$ as

$$
\begin{aligned}
F^{-, n}= & q f_{w}\left(s_{-1 / 2}^{n-1}\right)+h^{-\bar{\lambda}\left(s^{-, n-1}\right)} \\
& \cdot\left[J^{\prime}\left(s^{-, n-1}\right)\left(\frac{s^{-, n}-s_{-1 / 2}^{n}}{\Delta x / 2}\right)-\frac{h^{-} \tau}{\Delta x / 2}\left(\frac{s^{-, n}-s^{-, n-1}}{\Delta t}-\frac{s_{-1 / 2}^{n}-s_{-1 / 2}^{n-1}}{\Delta t}\right)\right], \\
F^{+, n}= & q f_{w}\left(s^{+, n-1}\right)+h^{+} \bar{\lambda}\left(s^{+, n-1}\right) \\
& \cdot\left[J^{\prime}\left(s^{+, n-1}\right)\left(\frac{s_{+1 / 2}^{n}-s^{+, n}}{\Delta x / 2}\right)-\frac{h^{+} \tau}{\Delta x / 2}\left(\frac{s_{+1 / 2}^{n}-s_{+1 / 2}^{n-1}}{\Delta t}-\frac{s^{+, n}-s^{+, n-1}}{\Delta t}\right)\right] .
\end{aligned}
$$

At the interface, we also define the left and right discretized pressures

$$
p^{+, n}=\frac{J\left(s^{+, n}\right)}{h^{+}}-\tau \frac{s^{+, n}-s^{+, n-1}}{\Delta t} \text { and } p^{-, n}=\frac{J\left(s^{-, n}\right)}{h^{-}}-\tau \frac{s^{-, n}-s^{-, n-1}}{\Delta t} .
$$

By using the extended pressure condition discussed before one has

$$
\left(p^{-, n}-p^{+, n}\right)\left(1-s^{+, n}\right)=0 .
$$

Defining

$$
g\left(s^{-, n}, s^{+, n}\right)=\frac{J\left(s^{-, n}\right)}{h^{-}}-\frac{J\left(s^{+, n}\right)}{h^{+}}-\tau \frac{s^{-, n}-s^{+, n}}{\Delta t},
$$

and

$$
C^{n-1}:=C\left(s^{-, n-1}, s^{+, n-1}\right)=\frac{\tau}{\Delta t}\left(s^{+, n-1}-s^{-, n-1}\right),
$$

(32) implies either $s^{+, n}=1$, or the pressure continuity

$$
g\left(s^{-, n}, s^{+, n}\right)-C\left(s^{-, n-1}, s^{+, n-1}\right)=0 .
$$

Obviously, $\partial_{1} g>0$ and $\partial_{2} g<0$. Further, given $s^{-} \in(0,1)$, one has

$$
\lim _{s^{+} \searrow 0} g\left(s^{-}, s^{+}\right)=-\infty,
$$

and

$$
g\left(s^{-}, 1\right)=\frac{J\left(s^{-}\right)}{h^{-}}-\frac{J(1)}{h^{+}}+\frac{\tau}{\Delta t}\left(1-s^{-}\right) \leq \frac{J(1)}{h^{-}}-\frac{J(1)}{h^{+}},
$$

since $g\left(s^{-}, \cdot\right)$ is strictly increasing and continuous. For any $C^{n-1} \in\left(-\infty, g\left(s^{-}, 1\right)\right]$, there exists a unique $s^{+}=s^{+}\left(s^{-}\right)$such that

$$
g\left(s^{-}, s^{+}\left(s^{-}\right)\right)=C^{n-1} .
$$


Also, note that $g\left(s^{-}, 1\right)$ is decreasing in $s^{-}$,

$$
\lim _{s^{-} \searrow 0} g\left(s^{-}, 1\right)=+\infty, \quad g(1,1)=\frac{J(1)}{h^{-}}-\frac{J(1)}{h^{+}}
$$

and therefore $g(\cdot, 1):(0,1] \rightarrow[g(1,1),+\infty)$ is one to one. Observe that if $c^{n-1}>g\left(s^{-}, 1\right)$, discretized pressure becomes discontinuous at the interface $x=0$. By (32), one obtains $s^{+n}=1$. In this way, we have actually constructed the curves in the $(0,1] \times(0,1]$ square:

$$
\begin{aligned}
& \text { if } C^{n-1}>g(1,1), \text { then } \Gamma\left(C^{n-1}\right)=\left\{\left(s^{-}, s^{+}\right) \mid s^{-} \in\left(0, D\left(C^{n-1}\right)\right], g\left(s^{-}, s^{+}\right)=C^{n-1}\right\} \\
& \cup\left\{\left(s^{-}, 1\right) \mid s^{-} \in\left(D\left(C^{n-1}\right), 1\right]\right\}, \\
& \text { if } C^{n-1} \leq g(1,1), \text { then } \Gamma\left(C^{n-1}\right)=\left\{\left(s^{-}, s^{+}\right) \mid s^{-} \in(0,1], g\left(s^{-}, s^{+}\right)=C^{n-1}\right\},
\end{aligned}
$$

where $D(\cdot)$ is the inverse of $g(\cdot, 1)$.

Below we give a property of the discretized extended pressure condition:

Proposition 6 If $\frac{\tau}{\Delta t}\left(s^{+, n-1}-s^{-, n-1}\right)>\frac{J(1)}{h^{-}}-\frac{J(1)}{h^{+}}$, then $\frac{\tau}{\Delta t}\left(s^{+, n}-s^{-, n}\right)>\frac{J(1)}{h^{-}}-\frac{J(1)}{h^{+}}$.

Proof If $p^{-, n} \neq p^{+, n}$, one has $s^{+, n}=1$, and obviously,

$$
\tau \frac{1-s^{-, n}}{\Delta t} \geq 0>\frac{J(1)}{h^{-}}-\frac{J(1)}{h^{+}} .
$$

If $p^{-, n}=p^{+, n}$, then we have

$$
\frac{J\left(s^{-, n}\right)}{h^{-}}-\frac{J\left(s^{+, n}\right)}{h^{+}}+\frac{\tau\left(s^{+, n}-s^{-, n}\right)}{\Delta t}=C^{n-1} \geq \frac{J(1)}{h^{-}}-\frac{J(1)}{h^{+}} .
$$

In this case, if $s^{+, n} \geq s^{-, n}$, one has

$$
\frac{\tau\left(s^{+, n}-s^{-, n}\right)}{\Delta t} \geq 0>\frac{J(1)}{h^{-}}-\frac{J(1)}{h^{+}},
$$

otherwise, $s^{+, n}<s^{-, n}$ implies

$$
\frac{J\left(s^{-, n}\right)}{h^{-}}-\frac{J\left(s^{+, n}\right)}{h^{+}}<0 .
$$

Together with (35), we yield

$$
\frac{\tau\left(s^{+, n}-s^{-, n}\right)}{\Delta t}>\frac{J(1)}{h^{-}}-\frac{J(1)}{h^{+}},
$$

which concludes the proof.

\subsection{Fully Implicit Scheme}

Here a nonlinear, implicit scheme is discussed as an alternative to the linear one. Next to improved stability properties, for this scheme we can prove that $s^{ \pm, n}$, the saturation at the interface, remain between 0 and 1 , a property that is not guaranteed for the linear scheme. To construct the scheme, we first define the decreasing function $\beta: \mathbb{R} \rightarrow \mathbb{R}$ by

$$
\beta(s)=\int_{0}^{s} \bar{\lambda}(z) J^{\prime}(z) \mathrm{d} z
$$


and rewrite the flux in (31) as

$$
F=q f_{w}(s)+h \frac{\partial \beta(s)}{\partial x}-\tau h^{2} \bar{\lambda}(s) \frac{\partial}{\partial x}\left(\frac{\partial s}{\partial t}\right) .
$$

As before, for $i \neq 0$ we write

$$
\frac{s_{i-1 / 2}^{n}-s_{i-1 / 2}^{n-1}}{\Delta t}=-\frac{F_{i}^{n}-F_{i-1}^{n}}{\Delta x},
$$

but now the upwind flux $F_{i}^{n}$ becomes

$$
\begin{aligned}
F_{i}^{n}= & q f_{w}\left(s_{i-1 / 2}^{n}\right)+\frac{h^{ \pm}}{\Delta x}\left(\beta\left(s_{i+1 / 2}^{n}\right)-\beta\left(s_{i-1 / 2}^{n}\right)\right) \\
& -\frac{\tau\left(h^{ \pm}\right)^{2}}{\Delta x \Delta t} \bar{\lambda}\left(\left(s_{i+1 / 2}^{n-1}+s_{i-1 / 2}^{n-1}\right) / 2\right)\left(\left(s_{i+1 / 2}^{n}-s_{i+1 / 2}^{n-1}\right)-\left(s_{i-1 / 2}^{n}-s_{i-1 / 2}^{n-1}\right)\right) .
\end{aligned}
$$

As before, by $h^{ \pm}$we mean $h^{-}$if $i<0$, or $h^{+}$if $i>0$. Further, if $i=0$, the flux is defined on each side of the interface as

$$
\begin{aligned}
F^{-, n}= & q f_{w}\left(s_{-\frac{1}{2}}^{n}\right)+\frac{2 h^{-}}{\Delta x}\left(\beta\left(s^{-, n}\right)-\beta\left(s_{-1 / 2}^{n}\right)\right) \\
& -\frac{2 \tau\left(h^{-}\right)^{2}}{\Delta x \Delta t} \bar{\lambda}\left(s^{-, n-1}\right)\left(\left(s^{-, n}-s^{-, n-1}\right)-\left(s_{-1 / 2}^{n}-s_{-1 / 2}^{n-1}\right)\right),
\end{aligned}
$$

and

$$
\begin{aligned}
F^{+, n}= & q f_{w}\left(s^{+, n}\right)+\frac{2 h^{+}}{\Delta x}\left(\beta\left(s_{+1 / 2}^{n}\right)-\beta\left(s^{+, n}\right)\right) \\
& -\frac{2 \tau\left(h^{+}\right)^{2}}{\Delta x \Delta t} \bar{\lambda}\left(s^{+, n-1}\right)\left(\left(s_{+1 / 2}^{n}-s_{+1 / 2}^{n-1}\right)-\left(s^{+, n}-s^{+, n-1}\right)\right) .
\end{aligned}
$$

For having a conservative scheme, the two expressions should be equal. Combined with the pressure condition (32), and viewing $s_{ \pm 1 / 2}^{n}$ as well as the saturation values $s_{i+1 / 2}^{n-1}$ and $s^{ \pm, n-1}$ as known, this provides a nonlinear system with $s^{ \pm, n}$ as unknowns. Below we show that this system has a unique solution pair in the square $[0,1]^{2}$.

The condition $F^{-, n}=F^{+, n}$ can be written as

$$
R\left(s^{-, n}, s^{+, n}\right)=B\left(s_{-1 / 2}^{n}, s_{+1 / 2}^{n}\right),
$$

where

$$
\begin{aligned}
R\left(s^{-, n}, s^{+, n}\right)= & q f_{w}\left(s^{+, n}\right)-\frac{2}{\Delta x}\left(h^{+} \beta\left(s^{+, n}\right)+h^{-} \beta\left(s^{-, n}\right)\right) \\
& +\frac{2 \tau}{\Delta x \Delta t}\left(\left(h^{+}\right)^{2} \bar{\lambda}\left(s^{+, n-1}\right) s^{+, n}+\left(h^{-}\right)^{2} \bar{\lambda}\left(s^{-, n-1}\right) s^{-, n}\right), \\
B= & q f_{w}\left(s_{-1 / 2}^{n}\right)-\frac{2}{\Delta x}\left(h^{-} \beta\left(s_{-1 / 2}^{n}\right)+h^{+} \beta\left(s_{+1 / 2}^{n}\right)\right) \\
& +\frac{2 \tau}{\Delta x \Delta t}\left(\left(h^{-}\right)^{2} \bar{\lambda}\left(s^{-, n-1}\right)\left(s^{-, n-1}+\left(s_{-1 / 2}^{n}-s_{-1 / 2}^{n-1}\right)\right)\right. \\
+ & \left.\left(h^{+}\right)^{2} \bar{\lambda}\left(s^{+, n-1}\right)\left(s^{+, n-1}+\left(s_{+1 / 2}^{n}-s_{+1 / 2}^{n-1}\right)\right)\right) .
\end{aligned}
$$


Using (36), $B$ becomes

$$
\begin{aligned}
B= & q f_{w}\left(s_{-1 / 2}^{n}\right)-\frac{2}{\Delta x}\left(h^{-} \beta\left(s_{-1 / 2}^{n}\right)+h^{+} \beta\left(s_{+1 / 2}^{n}\right)\right) \\
& +\frac{2 \tau}{\Delta x \Delta t}\left(\left(h^{-}\right)^{2} \bar{\lambda}\left(s^{-, n-1}\right)\left(s^{-, n-1}-\frac{\Delta t}{\Delta x}\left(F^{-, n}-F_{-1}^{n}\right)\right)\right. \\
& \left.+\left(h^{+}\right)^{2} \bar{\lambda}\left(s^{+, n-1}\right)\left(s^{+, n-1}-\frac{\Delta t}{\Delta x}\left(F_{1}^{n}-F^{+, n}\right)\right)\right) .
\end{aligned}
$$

Obviously, $R$ is increasing in both arguments and one has

$$
\begin{aligned}
R(0,0) & =0 \leq R\left(s^{-}, s^{+}\right) \leq R(1,1) \\
& =q-\frac{2}{\Delta x}\left(h^{+}+h^{-}\right) \beta(1)+\frac{2 \tau}{\Delta x \Delta t}\left[\left(h^{+}\right)^{2} \bar{\lambda}\left(s^{+, n-1}\right)+\left(h^{-}\right)^{2} \bar{\lambda}\left(s^{-, n-1}\right)\right] .
\end{aligned}
$$

Note that, if both $s^{-, n-1}, s^{+, n-1}$ take the values 0 or 1 , the last terms in (39) vanish, giving

$$
B=q f_{w}\left(s_{-1 / 2}^{n}\right)-\frac{2}{\Delta x}\left(h^{-} \beta\left(s_{-1 / 2}^{n}\right)+h^{+} \beta\left(s_{+1 / 2}^{n}\right)\right) .
$$

Since $\beta$ is decreasing, in this case one has $0 \leq B \leq R(1,1)$. Further, if $s^{-, n-1}$ is not equal to 0 or to 1 , with $\Delta t$ small enough one gets

$$
0 \leq s^{-, n-1}-\frac{\Delta t}{\Delta x}\left(F^{-, n}-F_{-1}^{n}\right) \leq 1,
$$

and analogously for $s^{+, n-1}$. From (39), this shows again that $0 \leq B \leq R(1,1)$.

This gives the following:

Lemma 1 For a sufficiently small time step $\Delta t$, with $C^{n-1}=C\left(s^{-, n-1}, s^{+, n-1}\right)$ defined in (33) and for $R$ and $B$ in (37) and (39), the system

$$
\begin{aligned}
& R\left(s^{-, n}, s^{+, n}\right)=B, \\
& \left(g\left(s^{-, n}, s^{+, n}\right)-C^{n-1}\right)\left(1-s^{+, n}\right)=0,
\end{aligned}
$$

has a unique solution pair $\left(s^{-, n}, s^{+, n}\right) \in[0,1]^{2}$.

Proof The set $\Gamma\left(C^{n-1}\right)$ introduced in (34) contains pairs satisfying the pressure condition (32). In this set, we seek a pair $\left(s_{0}^{-, n}, s_{0}^{+, n}\right)$ such that

$$
R\left(s_{0}^{-, n}, s_{0}^{+, n}\right)=B\left(s_{0}^{-, n}, s_{0}^{+, n}\right) .
$$

If such a pair exists, it solves the system (40). Since $g$ is increasing in the first argument and decreasing in the second one, as long as both $s^{-, n}$ and $s^{+, n}$ are below 1 , the curve $\Gamma\left(C^{n-1}\right)$ is a graph of a general non-decreasing function (see Fig. 9). Similarly, for $B \in[0, R(1,1)]$, the set $R(\cdot, \cdot)=B$ is the graph of a decreasing function in the $s^{-, n} s^{+, n}$-plane. Also, observe that both curves are continuous. Therefore, these two curves have at most one intersection point inside the square $[0,1]^{2}$, implying that (40) has a unique solution pair.

Note that if the solution pair provided above lies inside $[0,1)^{2}$, then one has pressure continuity across the interface, $p^{-, n}=p^{+, n}$. Otherwise, the solution pair lies on the boundary of the square $[0,1]^{2}$. Moreover, assuming that initially one has $s^{-, 0} \leq s^{+, 0}$, implying that at the interface separating the two blocks more oil is present at the coarse material side than at the fine material side, repeating the proof of Proposition 6 one obtains that $s^{-, n} \leq s^{+, n}$ 
Fig. 9 The curves $g(\cdot, \cdot)=C$ and $R(\cdot, \cdot)=B$

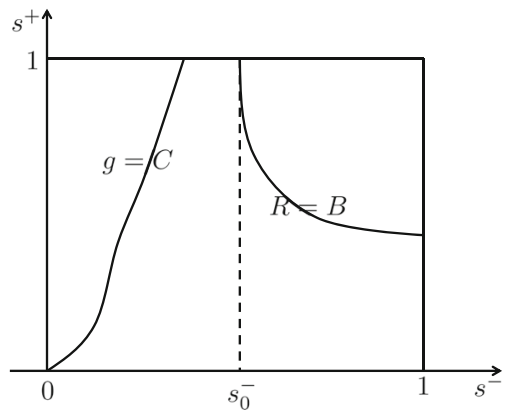

for all $n$. This means that pressure discontinuity at the interface can only occur if no oil is present at the fine material side, $s^{+, n}=1$, which is precisely the discrete pressure condition in (32).

Remark 6 The construction above assumes that $s_{-1 / 2}^{n}, s_{1 / 2}^{n}$ are known. In fact, these are part of the solution computed implicitly, at time step $t_{n}$. This means that, actually, $s_{-1 / 2}^{n}, s_{1 / 2}^{n}$ and consequently $B$ depend on $s^{-, n}, s^{+, n}$. However, decoupling the calculation of the solution pair $s^{ \pm, n}$ from the effective time stepping suggests an iterative procedure for the implicit scheme: using, say, the values $s^{ \pm, n-1}$ as starting point, compute $s_{i+1 / 2}^{n}$ by solving (36) away from the interface, and use $s_{-1 / 2}^{n}, s_{1 / 2}^{n}$ to update $s^{ \pm, n}$.

\subsection{Numerical Results}

In this section, we give the calculations for the model by using linear implicit method, which is much easier to implement than the fully implicit scheme in Sect. 4.2. Here, we used the following functions and parameters:

$$
k_{r w}(s)=s^{2}, k_{r n}(s)=(1-s)^{2}, J(s)=s^{-1}, N_{c}=1, M=1, h^{-}=1, h^{+}=0.5 .
$$

The tests are done in the interval $(-1,1)$. Further, we present the results in terms of the oil/non-wetting phase saturation $s_{o}=1-s$, as this is the phase for which trapping may occur. The initial oil saturation is hat shaped (see Fig. 10)

$$
s_{o}(x, 0)=s_{o}^{0}:= \begin{cases}0, & -1<x<-0.34, \\ 0.9, & -0.34 \leq x \leq-0.12 \\ 0, & -0.12<x<1 .\end{cases}
$$

At $x= \pm 1$ we take homogeneous boundary conditions, $\partial_{x} s( \pm 1, t)=0$. This mimics the situations when an oil blob in the coarse layer is displaced by water. After a certain time, the oil reaches the interface. Note that initially no oil is present in the fine medium.

Before discussing the results, we recall that the saturation $s^{*}$ defined in (14) is the limit saturation allowing for pressure continuity in the equilibrium models $(\tau=0)$. This also defines an entry saturation for the oil, $s^{\text {entry }}=1-s^{*}$. For the equilibrium model, oil flows into the fine material only if $s_{O}>s^{\text {entry }}$ at the coarse material side of the interface, and it remains trapped if $s_{o} \leq s^{\text {entry }}$. Figure 11, displaying the results obtained for $\tau=0$ at $t=0.7$, confirms this statement. At the coarse side of the interface, one has $s_{o}^{-}<s^{\text {entry }}$ (picture on the left) and the oil flux is 0 there (picture in the middle). This means that no oil enters into the fine medium. Further, the pressure is discontinuous at the interface (picture on the right). 
Fig. 10 Initial oil saturation $s_{o}^{0}$
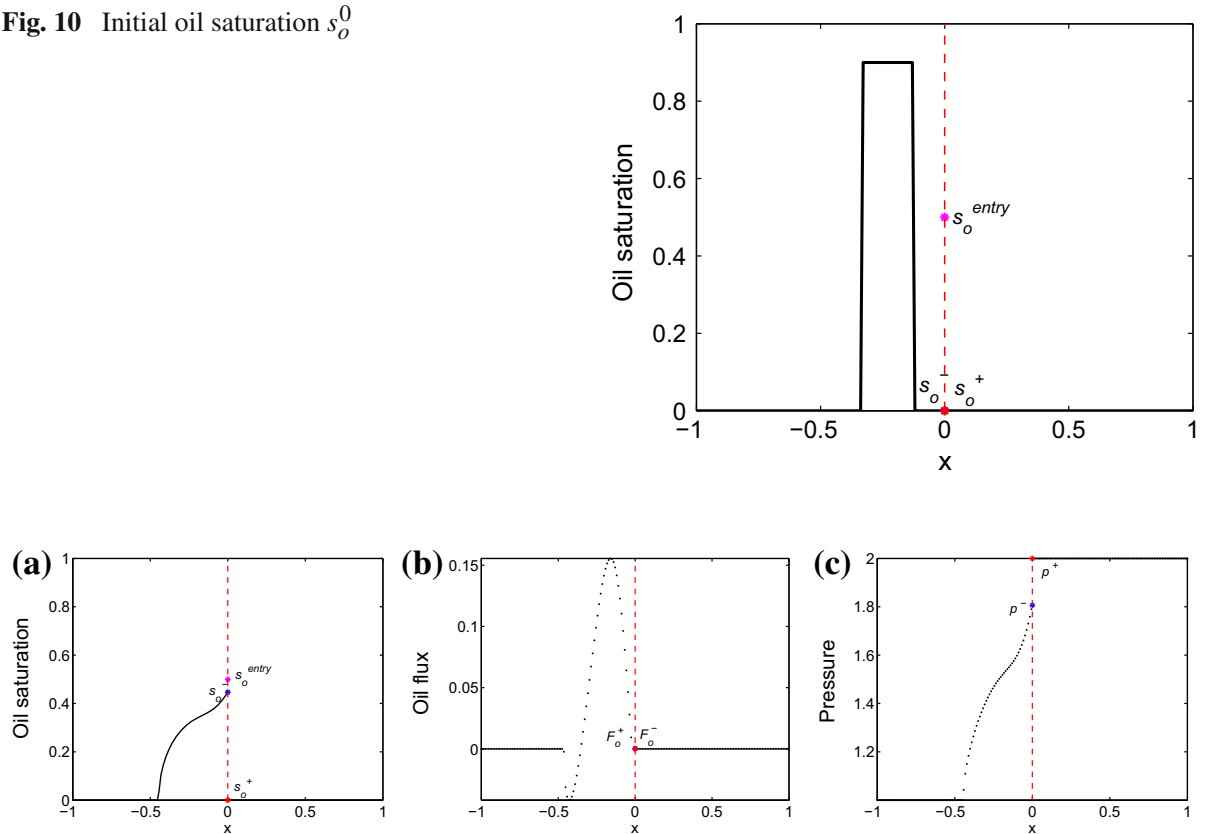

Fig. $11 \tau=0, t=0.7$ : oil saturation (a), oil flux (b) and phase pressure difference (c)
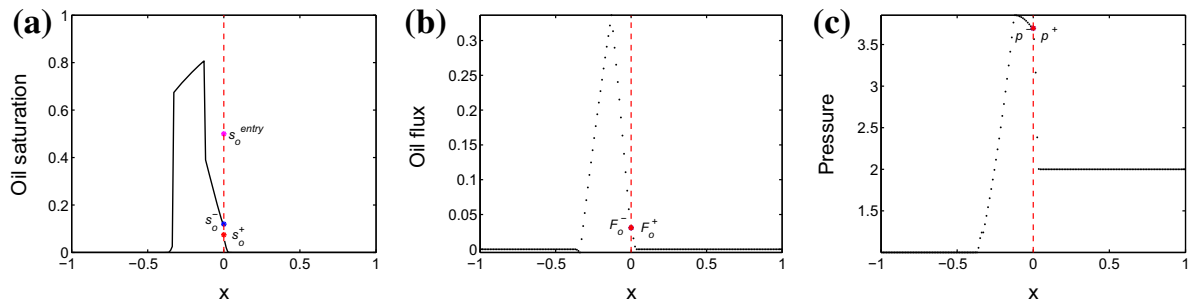

Fig. $12 \tau=1, t=0.7$ : oil saturation (a), oil flux (b) and phase pressure difference (c)

The case $\tau=1$, presented in Fig. 12, shows a different situation. In the left picture, although $s_{o}^{-}<s^{\text {entry }}$, one still has $s_{o}^{+}>0$, meaning that oil has already entered in the fine medium. This is also confirmed by the middle picture, displaying a nonzero oil flux at the interface. Finally, the picture on the right shows that the pressure is continuous.

Figure 13 presents the results for $\tau=0$ and at $t=4$. Then, oil has flown into the fine medium (picture on the left). The flux and the pressure are both continuous (middle and right pictures). At the same time, but with $\tau=1$, we observe that more oil has flown into the fine media (left picture of Fig. 14). As expected, the flux and pressure are continuous as well.

The results above suggest that the amount of oil flowing into the fine material increases with $\tau$. To understand this behavior, we compare the oil saturation obtained for $\tau=0, \tau=1$ and $\tau=10$, all at the same time $t=4$. The profiles in Fig. 15 show that, for $\tau=0$, little oil has flown into the fine media, and this amount is higher for $\tau=1$. In both cases, $s_{o}^{-}$, the oil saturation at the coarse side of the interface, already exceeds the entry saturation $s^{\text {entry }}$. However, for $\tau=10, s_{o}^{-}<s^{\text {entry }}$, but oil has still flown into the fine material. One expects 

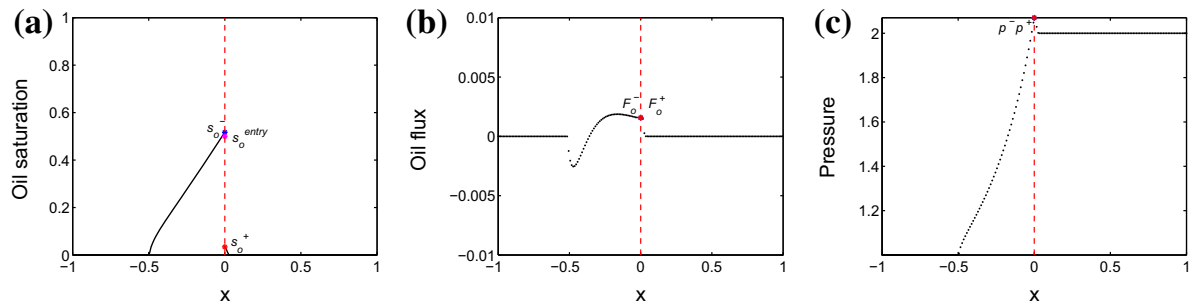

Fig. $13 \tau=0, t=4$ : oil saturation (a), oil flux (b) and phase pressure difference (c)
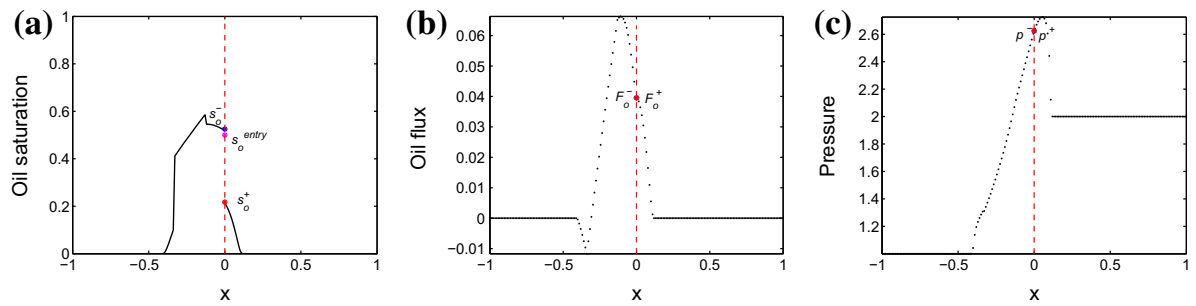

Fig. $14 \tau=1, t=4$ : oil saturation (a), oil flux (b) and phase pressure difference (c)
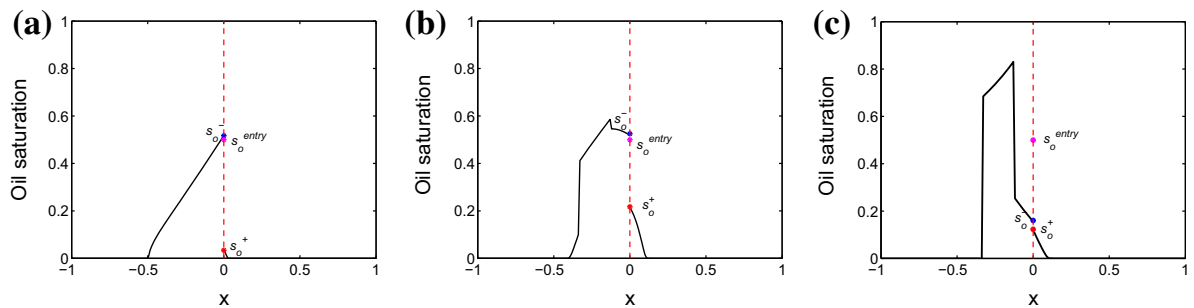

Fig. $15 t=4$, oil saturation: $\tau=0(\mathbf{a}), \tau=1(\mathbf{b})$ and $\tau=10(\mathbf{c})$

that the oil flow into the fine material will take longer for the largest value of $\tau$, in agreement with Corollary 1.

Finally, we observe that in the equilibrium case $\tau=0$ one can determine the maximal amount of oil that can be trapped at the interface, see Duijn et al. (1995). Having this in mind, we choose again a hat-shaped initial saturation

$$
s_{o}(x, 0)=s_{o}^{0}:= \begin{cases}0, & -1<x<-0.34, \\ 0.4, & -0.34 \leq x \leq-0.12 \\ 0, & -0.12<x<1,\end{cases}
$$

where the total amount of oil equals the maximal amount that can be trapped for equilibrium models. With this initial data, we compute the numerical solutions for three values of $\tau$, namely 0,10 and 30 . Figure 16 shows the results at $t=400$, when practically all solutions have reached a steady state and no oil flow is encountered anymore.

The left picture shows the result for $\tau=0$. In this case, the entire amount of oil is trapped in the coarse medium, and the oil saturation $s_{o}^{-}$matches the entry saturation $s^{\text {entry }}$. No oil has flown at all into the fine material. As following from the middle picture, for $\tau=10$, one has $s_{o}^{-}<s^{\text {entry }}$, and the oil remaining trapped in the coarse material is less than in the equilibrium 

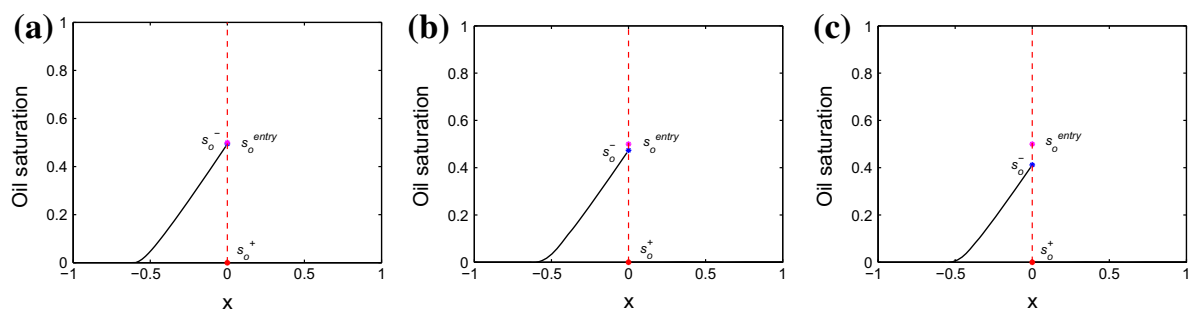

Fig. $16 t=400$, oil saturation: $\tau=0(\mathbf{a}), \tau=10(\mathbf{b})$ and $\tau=30(\mathbf{c})$

case. The situation becomes more obvious for the solution corresponding to $\tau=30$. The oil saturation $s_{o}^{-}$has decayed further, and the trapped oil is less than in the previous cases. This is again in agreement with the analysis in Sect. 3.2.

\section{Conclusions}

We have considered a non-equilibrium model for two-phase flow in heterogeneous porous media, where dynamic effects are included in the phase pressure difference. A simple situation is considered, where the medium consists of two adjacent homogeneous blocks. We obtain the conditions coupling the models in each of the two sub-domains. The first condition is, as expected, flux continuity, whereas the second is an extended pressure condition extending the results in Duijn and Neef (1998) for the standard two-phase flow model.

In the equilibrium case, if an entry pressure model is considered, oil can flow into the fine material only if its saturation exceeds an entry point. In the non-equilibrium case instead, the non-wetting phase may flow even if the oil saturation at the coarse side of the interface is below the entry point, and amount of oil remaining trapped at the interface is less than in the case of equilibrium models.

Finally, two different numerical schemes are discussed, and different numerical experiments are presented to sustain the theoretical findings.

Acknowledgments The work of X. Cao is supported by CSC (China Scholarship Council). The work of C.J. van Duijn and I.S. Pop is supported by the Shell-NWO/FOM CSER programme (project 14CSER016). Both supports are gratefully acknowledged. We thank Prof. Dr.-Ing. R. Helmig and I. Zizina (both Stuttgart) for the helpful discussions. We also thank the anonymous referees for their valuable suggestions. The authors are members of the International Research Training Group NUPUS funded by the German Research Foundation DFG (GRK 1398), the Netherlands Organization for Scientific Research NWO (DN 81-754) and by the Research Council of Norway (215627).

Open Access This article is distributed under the terms of the Creative Commons Attribution 4.0 International License (http://creativecommons.org/licenses/by/4.0/), which permits unrestricted use, distribution, and reproduction in any medium, provided you give appropriate credit to the original author(s) and the source, provide a link to the Creative Commons license, and indicate if changes were made.

\section{References}

Andreianov, B., Cancès, C.: A phase-by-phase upstream scheme that converges to the vanishing capillarity solution for countercurrent two-phase flow in two-rocks media. Comput. Geosci. 18, 211-226 (2014)

Bertsch, M., Dal Passo, R., van Duijn, C.J.: Analysis of oil trapping in porous media. SIAM J. Math. Anal. 35, 245-267 (2003) 
Bottero, S.: Advances in the theory of capillarity in porous media. Ph.D. Thesis, University of Utrecht (2009)

Brenner, K., Cancès, C., Hilhorst, D.: Finite volume approximation for an immiscible two-phase flow in porous media with discontinuous capillary pressure. Comput. Geosci. 17, 573-597 (2013)

Buzzi, F., Lenzinger, M., Schweizer, B.: Interface conditions for degenerate two-phase flow equations in one space dimension. Analysis 29, 299-316 (2009)

Cancès, C., Pierre, M.: An existence result for multidimensional immiscible two-phase flows with discontinuous capillary pressure field. SIAM J. Math. Anal. 44, 966-992 (2012)

Cao, X., Pop, I.S.: Uniqueness of weak solutions for a pseudo-parabolic equation modeling two phase flow in porous media. Appl. Math. Lett. 46, 25-30 (2015)

Cuesta, C.M., Pop, I.S.: Numerical schemes for a pseudo-parabolic Burgers equation: discontinuous data and long-time behaviour. J. Comput. Appl. Math. 224, 269-283 (2009)

DiCarlo, D.A.: Experimental measurements of saturation overshoot on infiltration. Water Resour. Res. 40, W04215.1-W04215 (2004)

Fan, Y., Pop, I.S.: A class of degenerate pseudo-parabolic equations: existence, uniqueness of weak solutions, and error estimates for the Euler-implicit discretization. Math. Methods Appl. Sci. 34, 2329-2339 (2011)

Fučík, R., Mikyška, J.: Discontinous Galerkin and Mixed-Hybrid Finite Element Approach to Two-Phase Flow in Heterogeneous Porous Media with Different Capillary Pressures. Procedia Comput. Sci. 4, 908-917 (2011)

Fučík, R., Mikyška, J.: Mixed-hybrid finite element method for modelling two-phase flow in porous media. J. Math-for-Industry 3, 919 (2011)

Hassanizadeh, S.M., Gray, W.G.: Thermodynamic basis of capillary pressure in porous media. Water Resour. Res. 29, 3389-3405 (1993)

Helmig, R.: Multiphase Flow and Transport Processes in the Subsurface: A Contribution on the Modeling of Hydrosystems. Springer, Berlin (1997)

Helmig, R., Weiss, A., Wohlmuth, B.I.: Dynamic capillary effects in heterogeneous porous media. Comput. Geosci. 11, 261-274 (2007)

Helmig, R., Weiss, A., Wohlmuth, B.I.: Variational inequalities for modeling flow in heterogeneous porous media with entry pressure. Comput. Geosci. 13, 373-389 (2009)

Mikelić, A.: A global existence result for the equations describing unsaturated flow in porous media with dynamic capillary pressure. J. Differ. Equ. 248, 1561-1577 (2010)

Nordbotten, J.M., Celia, M.A.: Geological Storage of CO2: Modeling Approaches for Large-Scale Simulation. Wiley, New Jersey (2012)

Radu, F.A.: Mixed finite element discretization of Richards equation: error analysis and application to realistic infiltration problems. Ph.D. Thesis, University of Erlangen-Nürnberg (2004)

Radu, F.A., Nordbotten, J.M., Pop, I.S., Kumar, K.: A robust linearization scheme for finite volume based discretizations for simulation of two-phase flow in porous media. J. Comput. Appl. Math. 289, 134-141 (2015)

van Duijn, C.J., Molenaar, J., de Neef, M.J.: The effect of capillary forces on immiscible two-phase flow in heterogeneous porous media. Transp. Porous Media 21, 71-93 (1995)

van Duijn, C.J., Mikelić, A., Pop, I.S.: Effective equations for two-phase flow with trapping on the micro scale. SIAM J. Appl. Math. 62, 1531-1568 (2002)

van Duijn, C.J., Eichel, H., Helmig, R., Pop, I.S.: Effective equations for two-phase flow in porous media: the effect of trapping on the microscale. Transp. Porous Media 69, 411-428 (2007)

van Duijn, C.J., Peletier, L.A., Pop, I.S.: A new class of entropy solutions of the Buckley-Leverett equation. SIAM J. Math. Anal. 39, 507-536 (2007)

van Duijn, C.J., Fan, Y., Peletier, L.A., Pop, I.S.: Traveling wave solutions for degenerate pseudo-parabolic equation two-phase flow in porous media. Nolinear Anal. Real World Appl. 14, 1361-1383 (2013)

van Duijn, C.J., de Neef, M.J.: Similarity solution for capillary redistribution of two phases in a porous medium with a single discontinuity. Adv. Water. Resour. 21, 451-461 (1998) 